


\title{
Nervous system involvement in clinical peripheral inflammation: A description of three pediatric cases
}

\author{
Giovanna Vitaliti, Omidreza Tabatabaie', Nassim Matin', Giovanni Roberto Giugno², \\ Piero Pavone, Riccardo Lubrano ${ }^{3}$, Raffaele Falsaperla
}

Department of General Paediatrics and Neonates, Paediatric Operative Unit and Acute and Emergency, Vittorio Emanuele University Hospital, University of Catania, Catania, ${ }^{2}$ Department of General Paediatrics and Neonates, Paediatric and Neonatal Operative Unit, Gravina Hospital, Caltagirone, ${ }^{3}$ Pediatric Department, Pediatric Nephrology Operative Unit, La Sapienza University of Rome, Rome, Italy, ${ }^{1}$ Department of General Paediatrics and Neonates, Tehran University of Medical Sciences, Tehran, Iran

Address for correspondence: Dr. Giovanna Vitaliti, AOU Policlinico-OVE, Via Plebiscito n. 628, 95100 Catania, Italy. E-mail: giovitaliti@yahoo.it

\begin{abstract}
Latest research data have emphasized the interaction between the nervous and the immune systems. In this regard, it has been demonstrated that the disruption of the blood-brain barrier (BBB) secondary to peripheral inflammation may play a key role in this relationship. This assumption is linked to recent findings according to which units that constitute the BBB are not only simply neurologic but have also been reconsidered as "neurovascular" elements, through which immune system molecules are vehiculated within the central nervous system (CNS). Herein, we report two cases of food allergy (FA) and one case of infective gastroenteritis, associated with a spectrum of neurologic disorders involving both the CNS and the peripheral nervous system (PNS), postulating some etiopathogenic hypotheses to explain the link between peripheral inflammation and diseases of the nervous system (NS). Three pediatric cases of secondary NS involvement after gastrointestinal (GI) inflammation of different nature have been reported. The first case highlights the link between FA and CNS; the second one is based on a description of a link between $\mathrm{Gl}$ infection and CNS involvement while the third one describes the relationship between FA and PNS. The importance of these reports relies on the clinical demonstration of a link between the immune system and the NS. The relationship between immune system and NS seems to have pleiotropic aspects, involving different areas of the NS, such as CNS and PNS, which also seem to be in some way interconnected.
\end{abstract}

Key words: Case series, immune system, nervous system, pediatric, peripheral inflammation

\section{Introduction}

Latest research data have emphasized the interaction between the nervous system (NS) and the immune system. In regards, it has been demonstrated that the disruption

\begin{tabular}{|l|l|}
\hline \multicolumn{2}{|c|}{ Access this article online } \\
\hline Quick Response Code: & Website: \\
\cline { 1 - 2 } & www.pediatricneurosciences.com \\
& \\
& \\
\end{tabular}

of the blood-brain barrier (BBB) secondary to peripheral inflammation may play a key role in the explication of this relationship. This assumption is linked to recent findings according to which those units that constitute the BBB

\footnotetext{
This is an open access article distributed under the terms of the Creative Commons Attribution-NonCommercial-ShareAlike 3.0 License, which allows others to remix, tweak, and build upon the work non-commercially, as long as the
} author is credited and the new creations are licensed under the identical terms.

For reprints contact: reprints@medknow.com

Cite this article as: Vitaliti G, Tabatabaie O, Matin N, Giugno GR, Pavone P, Lubrano R, et al. Nervous system involvement in clinical peripheral inflammation: A description of three pediatric cases. J Pediatr Neurosci 2016;11:277-81. 
are not only simple neurologic units but have also been reconsidered as "neurovascular" elements, through which immune system molecules are vehiculated within the central nervous system (CNS).

Under a clinical point of view, several authors have tried to demonstrate this link, describing the CNS involvement in atopy, ${ }^{[1-7]}$ highlighting a clinical correlation between seizures and allergy to both inhalants and food.

Riazi et al. ${ }^{[8]}$ also showed, in murine models, an example of relationship between peripheral inflammation, phenotypically expressed as colitis, and seizures.

In addition, some reports in murine experimental models have shown a link also with the parasympathetic nervous system (PNS), confirming that inflammation involves NS in a pleiotropic fashion.

Herein, we report two cases of food allergy (FA) and one case of infective gastroenteritis, associated with a spectrum of neurologic disorders involving both the CNS and the PNS, postulating some etiopathogenic hypotheses to explain the link between peripheral inflammation and diseases of the NS.

\section{Case Reports}

\section{Case 1}

A 40-day-old infant was admitted to our Pediatric Operative Unit (OU), Vittorio Emanuele Hospital, University of Catania, Italy, due to frequent nausea and vomiting, followed by generalized clonic movements and loss of consciousness. Each episode took about 5-10 min.

The infant was delivered through cesarean section as product of a twin pregnancy, in which the other fetus had died in utero during the $8^{\text {th }}$ week of pregnancy. He was the second son of nonconsanguineous parents. At birth, he had been small for gestational age (weight $1800 \mathrm{~g}$ and height $48 \mathrm{~cm}$ ) and was admitted to the Neonatal Intensive Care Unit Department of a nearby hospital.

When admitted to our OU on the $40^{\text {th }}$ day of birth, his physical examinations were in favor of growth failure; weight and height were both below the $3^{\text {rd }}$ percentile $(3100 \mathrm{~g}$ and $49 \mathrm{~cm}$, respectively), and his head circumference was $36 \mathrm{~cm}$ $\left(3^{\text {rd }}-10^{\text {th }}\right.$ percentile). He had generalized muscle hypertonia and hyperreflexia, interrupted by generalized hypotonia.

A respiratory acidosis was detected at admission based on his venous blood gas ( $\mathrm{pH}: 7.31 ; \mathrm{PCO}_{2}: 38 \mathrm{mmHg} ; \mathrm{PO}_{2}: 51 \mathrm{mmHg}$; transcutaneous $\mathrm{O}_{2}$ saturation: $\left.100 \% ; \mathrm{HCO}_{3}{ }^{-}: 19.2 \mathrm{mmol} / \mathrm{l}\right)$. Except for a slight anemia ( $\mathrm{Hb}: 10.3 \mathrm{~g} / \mathrm{dl})$, his laboratory data were unremarkable; metabolic tests, serum levels of electrolytes, lactic acid, and bicarbonate were all within normal range.
During the hospitalization, it was noticed that episodes of vomiting, leg tremors, hypertonia, retropulsion of ocular globes, and clonic movements of the inferior lip after being fed with cow's milk.

The infant went under video electroencephalogram (EEG), showing a burst suppression-like pattern bit paroxysmal elements in bitemporal regions.

Cerebral magnetic resonance imaging (MRI) and cardiac ultrasound were all normal. Consultation with ophthalmologist and allergy specialist, and skin prick test (SPT) for cow's milk protein were unremarkable. A median large shadow of timic nature and marked bronchovascular trauma were noted in his chest X-ray. Slight congenital left kidney hydronephrosis was also noted in his abdomen ultrasound.

An amino acid infant formula free of cow's milk proteins (Neocate, Nestle) was prescribed for him that resulted in resolution of his neurological symptoms, normal arterial blood gas. The video EEG became normal within 2 weeks.

One month later, he went under a cow's milk protein challenge test that was according to the standard international protocols. ${ }^{[9]}$ The test was positive; the infant experienced diarrhea and vomiting, followed by suction movements at $150 \mathrm{mg}$ of cow's milk. Simultaneously, he was being monitored with EEG that showed generalized slowing of basal activity between 35 and $70 \mathrm{mg}$ of cow's milk. As a result, the infant was diagnosed with cow's milk allergy (CMA) and the amino acid infant formula was prescribed for him.

At the follow-up visit, a catch-up in growth was noticed (his growth parameters reached to the $25^{\text {th }}-50^{\text {th }}$ percentile) and he had not experienced further episodes of gastrointestinal (GI) and neurological symptoms.

\section{Case 2}

A 10-year-old Caucasian boy was admitted to Gravina and San Pietro Hospital of Caltagirone, Catania, Italy, for acute bronchitis, gastroenteritis, and the first episode of seizure.

The child was the third-born of nonconsanguineous parents, born by cesarean section, adequate for gestational age.

His familial anamnesis was negative for major diseases and only his mother was affected by inhalants allergy.

His personal anamnesis was negative for major diseases, and his developmental milestones had been reached at normal stages.

When admitted to hospital, the child presented with fever and diarrhea while his parents reported that 2 days before hospitalization, the patient presented a seizure characterized by skin paleness, generalized hypertonia, steadiness, followed 
by lethargy, which lasted some minutes, spontaneously resolved.

His physical examination at admission showed the presence of laterocervical and inguinal adenopathy, hyperemic pharynges, hypertrophic tonsils, decrease of vesicular murmur in his pulmonary basal left region, associated with crackles and normal neurological examination.

During hospitalization, the child underwent routine blood analyses, resulted within the normal range, except for total immunoglobulin E (IgE) (1804 UI/L; normal range: 70-100 UI/L) and increased value of polymerase chain reaction (as inflammation index) up to $10 \mathrm{mg} / \mathrm{dl}$. Serological examinations, urine and stool tests, and autoimmune examinations were within the normal range.

The child also underwent oculist and cardiologic consults that were normal. He has also performed an EEG study, which showed only a slight asymmetry, with a background alpha band activity more organized on his left side.

SPTs for inhalants were also performed, and they resulted positive for Dermatophagoides.

Antibiotic therapy (intravenous [i.v.] sulbactam and ampicillin, oral clarithromycin) and i.v. rehydration were performed during hospitalization with improvement of his symptoms.

At follow-up visits, after the resolution of his gastroenteritis, his parents referred that the child was completely restored and neither other neurologic symptoms nor seizures have been further observed.

\section{Case 3}

A 5-year-old female child was admitted to our OU for cyclic vomit, recurring with a constant frequency every 2 months, preceded by objective torque vertigo, headache, and weakness since she was 3 years old.

The child was the second-born of nonconsanguineous parents, born on term by cesarean section, adequate for gestational age.

Her familial anamnesis showed a high prevalence of allergy among her parents (her mother and father suffered of inhaled allergy) and her brother (who suffered of both inhaled and FA).

At physical examination at admission, her auxological parameters were within the normal range and her neurologic examination was normal. Cardiac, respiratory, and abdomen examinations were normal.

Routine blood analyses were within the normal range, except for total IgE dosage that was slightly high (340 UI/L, normal value: $70-100 \mathrm{UI} / \mathrm{L})$.
For the persistence of her symptoms, we performed a cardiac evaluation by Doppler echocardiogram and tilt test, a neurologic examination by EEG and cerebral MRI, and an ophthalmologic and ENT consulting, including visual and auditory evocative potentials. All the examinations were normal.

The child was therefore dismissed with a diagnosis of cyclic vomit.

Nevertheless, her symptoms persisted associated with recurrent abdominal pain and the child was therefore readmitted 8 months later her first hospitalization.

Routine blood analysis and blood gas analysis were still within the normal range. During this last hospitalization, the child underwent a specialist consult for allergy, performing SPTs for inhaled and food allergens, which gave a slight positivity for casein and tomato (the diameter of her wheal was $3.5 \mathrm{~mm}$ ). Therefore, she also underwent a radio allergo sorbent test (RAST) evaluation of food allergens, which confirmed the positive results found at SPTs (casein $>3.45 \mathrm{UI} / \mathrm{ml}$ and tomato $>3.80 \mathrm{UI} / \mathrm{ml}$ ). Considering these results, the child started a casein and tomato-free diet with complete resolution of her symptoms.

At follow-up visit, she did not refer other cyclic vomit episodes, headache, or weakness, and she is actually following a phase of reintroduction of casein and tomato, according to the standard desensibilization protocols, ${ }^{[9]}$ without further neurologic symptoms.

\section{Discussion}

Herein, we report three cases of peripheral inflammation with secondary NS involvement. The first case highlights the link between FA and CNS; the second one is based on a description of a link between GI infection and CNS involvement while the third one describes the relationship between FA and parasympathetic NS (PNS).

The first case represents an example of CNS secondary involvement in a patient affected by FA. The peculiarity of this case relies on the unusual clinical association between CMA and epilepsy. In regards, Falsaperla et al. and Vitaliti have been the first authors postulating an etiopathogenic link to explain this clinical association. ${ }^{[10]}$ According to the Falsaperla-Vitaliti's hypothesis, a peripheral inflammation involving the GI tract is responsible for activation of the GI immune system, with consequent allergen-mediated stimulation of local antigen-presenting cells, type 2 helper T (Th2)-lymphocytes subsets, and secretion of pro-inflammatory cytokines. The local inflammation would then cause a serum diffusion of pro-inflammatory cytokines to the $\mathrm{BBB}$, causing $\mathrm{BBB}$ mast cells activation and secondary induction of T-lymphocytes, with consequent onset of BBB disruption and CNS inflammation, triggering seizures. On 
the other hand, milk proteins in CMA patients cause a GI inflammation responsible for the induction of T-lymphocytes sensitized to cow's milk protein and migration of the same sensitized lymphocytes within the BBB, as a consequence of a process of "homing." ${ }^{[11]}$ Therefore, the passage of cow's milk protein through the GI-inflamed mucosa in the systemic circulation may be responsible for secondary activation of sensitized T-lymphocytes within the BBB and induction of the inflammation cascade within the CNS.

Another important consideration rising from the first presented case is that the child was born from a twin pregnancy, in which his brother died at 8 weeks of gestation. This event may have caused an immune dysregulation toward enhancement of pro-inflammatory processes and production of oxidative stress that leads to intrauterine growth restriction. These intrauterine events might be associated to $\mathrm{BBB}$ vulnerability, according which pro-inflammatory stimuli, causing diseases at further ages, in this case have been responsible for an earlier presentation of symptoms. This according to a deep clinical improvement at follow-up visits performed after 12 months of age.

The second case describes a larger example of peripheral inflammation, including both gastroenteritis and allergic diseases to inhalants. In regards, the link between gut inflammation and BBB disruption has recently been studied by Riazi et al. ${ }^{\left[{ }^{[8]}\right.}$ who showed how peripheral inflammation alters hippocampal neuronal excitability, studying a murine model of 2,4,6-trinitrobenzene sulfonic acid-induced colitis. TNBS caused a Th1-cell-mediated bowel inflammation with consequent activation of the humoral immune system. The authors identified a model of microglial-dependent, tumor necrosis factor (TNF)-mediated increase in CNS excitability that may be considered a potential link between peripheral and central inflammation. These data were confirmed by the same authors through a reversible increase in seizure susceptibility, directly correlating with the grade of severity of inflammation and hippocampal electrophysiological recordings of inflamed mice. These findings revealed increased excitability and epileptiform burst discharges to bath application of potassium channel blocker 4-AP, as expressed by increased excitability of neuronal networks in the hippocampus following peripheral inflammation, suggesting that the production of TNF-a occurs within the brain during peripheral inflammation, increasing seizure susceptibility. Moreover, increased TNF-signaling in the CNS as well as microglial activation appeared sufficient for changes in seizure susceptibility. The immune mechanism of epileptogenesis secondary to peripheral inflammation has centered upon the role of microglia. Microglial activation as a consequence of peripheral inflammation was confirmed by the increased number of microglial cells in the hippocampus and adjacent areas. ${ }^{[12-14]}$ In keeping with this observation, the authors showed an increased production of hippocampal TNF-a, despite low interleukin-1 (IL-1) levels, explaining that these findings were likely related to the colonic inflammatory model, which at the height of colic inflammation is associated with a downregulation of IL-1 synthesis in comparison with
TNF-a secretion. These findings have been supported by the literature implicating pro-inflammatory cytokines in seizure activity and epilepsy, ${ }^{[15-18]}$ with a predominant role for IL-1b as a proconvulsing cytokine and IL-1Ra, mainly playing an anticonvulsant role. ${ }^{[15-18]}$

The third case is an example of association between FA and secondary involvement of parasympathetic nervous system (PNS). This case differs from the first one because FA realizes with an IgE-dependent mechanism, linked to a positivity of food allergens SPT and RAST, while in the first case the pathogenic mechanism was non- $\operatorname{IgE}$ mediated. This is a novel literature finding as the only clinical report in childhood on the association between allergy and PNS involvement has been described by Wu and Gao, ${ }^{[19]}$ in a 13-year-old male child with frequent episodes of vertigo and otologic symptoms. In this case, a Ménière's disease was diagnosed but failed to respond to conventional treatment. Allergy testing revealed serious reactions to many allergens, and autonomic tests showed that he was dysautonomic. An allergen-restricted diet and treatment of dysautonomia were effective, the boy being free from vertigo within 2 months. The authors therefore concluded that the autonomic nerves and the immune system could interact. ${ }^{[19]}$

On a laboratory point of view, immunochemistry data have demonstrated the expression of $\operatorname{IgE}$ receptor on the vagus nerve in the mouse intestine. In regards, Liang et al., in 2011, isolated vagus ganglion neurons (VGN) from mice and cultured in vitro. They examined the $\mathrm{IgE}$ receptor/ $\operatorname{IgE}$ complex on VGN in mouse models of FA, evaluating the effect of the partial removal of the vagal nerve via surgery or administration with anticholinergic agents on the suppression of Th2 inflammation. ${ }^{[20]}$ The authors found a high-affinity IgE receptor on the intestinal vagus nerve and an increased expression of the $\mathrm{IgE}$ receptor on the vagus nerve in the intestines of mice with intestinal immune inflammation. Isolated mouse VGN expressed IgE receptor I, which could form complexes with IgE. Re-exposure to specific antigens activated the sensitized VGN, manifesting the release of transmitter glutamate that could activate dendritic cells by increasing the expression of CD80 and major compatibility complex class II and suppressing IL-12. The partial removal of the vagus nervous suppressed Th2 inflammation in the intestine. ${ }^{[20]}$ Therefore, authors concluded that the mice intestinal vagus nerve expressed a high-affinity IgE receptor, underlying the relationship between enteric inflammation and activation of the parasympathetic nervous system.

More recently, in 2014, Yamamoto et al. ${ }^{[21]}$ investigated in murine models the cholinergic anti-inflammatory pathway as a regulatory system to ameliorate-disrupted mucosal immune homeostasis in the gut. BALB/c mice sensitized with ovalbumin received repeated oral ovalbumin to determine the onset of FA. FA mice developed severe allergic diarrhea and exhibited enhanced Th2 cell immune responses in both systemic immunity and mucosal immunity, along with mucosal mast cells hyperplasia in the colon. However, these symptoms 
did not develop in p85a disrupted phosphoinositide-3 kinase-deficient mice that lacked mast cells in the gut. Vagal stimulation by 2 -deoxy-D-glucose and drug treatment with nicotinic ACh receptor (nAChR) agonists (nicotine and a7 nAChR agonist GTS-21) alleviated the allergic symptoms in the FA mice. Nicotine treatment suppressed mucosal mast cells hyperplasia and upregulated mRNA expression of Th1 and Th2 cytokines in the FA mice colon. Mucosal mast cells, which were negatively regulated by a7-nAChRs, were often located in proximity to cholinergic calcitonin gene-related peptide immunoreactive nerve fibers in the FA mice colon. Therefore, the authors concluded that the cholinergic neuroimmune interaction via a $7-n A C h R s$ on mucosal mast cells is largely involved in maintaining intestinal immune homeostasis and can be a target for a new therapy against mucosal immune diseases with homeostatic disturbances such as FA. ${ }^{[21]}$

Taken together, these studies highlight the relationship between immune system and parasympathetic nervous system, opening a new research scenario on the link between PNS and immune system, possibly targeting the PNS for those clinical diseases involving the two systems. Moreover, the authors thought that this relationship is of relevant clinical importance as in case of autonomic symptoms such as vertigo or any vagal disease; healthcare assistants should also exclude the presence of allergy.

\section{Conclusions}

The importance of these reports relies in the clinical demonstration of a link between the immune system and the NS, and this relationship seems to have pleiotropic aspects, involving different areas of the NS, such as the CNS and the PNS, which also seem to be in some way interconnected. This link is actually object of new literature data to clarify those pathogenic aspects subtended in this relationship and to target new therapeutic strategies to improve the treatment efficacy of emerging neurologic challenges.

\section{Financial support and sponsorship}

Nil.

\section{Conflicts of interest}

There are no conflicts of interest.

\section{References}

1. Spangler RH. Some allergic factors in essential epilepsy. J Allergy 1931;3:39-50.
2. Gray PJ. Epilepsy and allergy. Br Med J (Clin Res Ed) 1981;283:231.

3. Pavone P, Longo MR, Scalia F, Polosa R, Kira J, Falsaperla R. Recurrent Hopkin's syndrome: A case report and review of the literature. J Neurol Sci 2010;297:89-91.

4. Castaneda GY, Heilbroner PL, Shah N, Forem S, Fish I. Asthma and epilepsy: Are they related? A retrospective study. J Child Neurol 1998;13:283-5.

5. Pelliccia A, Lucarelli S, Frediani T, D’Ambrini G, Cerminara C, Barbato M, et al. Partial cryptogenetic epilepsy and food allergy/ intolerance. A causal or a chance relationship? Reflections on three clinical cases. Minerva Pediatr 1999;51:153-7.

6. Frediani T, Pelliccia A, Aprile A, Ferri E, Lucarelli S. Partial idiopathic epilepsy: Recovery after allergen-free diet. Pediatr Med Chir 2004;26:196-7.

7. Lucarelli S, Spalice A, D'Alfonso Y, Lastrucci G, Sodano S, Topazio L, et al. Cow's milk allergy and rolandic epilepsy: A close relationship? Arch Dis Child 2012;97:481.

8. Riazi K, Galic MA, Pittman QJ. Contributions of peripheral inflammation to seizure susceptibility: Cytokines and brain excitability. Epilepsy Res 2010;89:34-42.

9. Fiocchi A, Schünemann HJ, Brozek J, Restani P, Beyer K, Troncone R, et al. Diagnosis and rationale for action against cow's milk allergy (DRACMA): A summary report. J Allergy Clin Immunol 2010;126:1119-28.e12.

10. Falsaperla R, Pavone P, Miceli Sopo S, Mahmood F, Scalia F, Corsello G, et al. Epileptic seizures as a manifestation of cow's milk allergy: A studied relationship and description of our pediatric experience. Expert Rev Clin Immunol 2014;10:1597-609.

11. Geginat J, Paroni M, Maglie S, Alfen JS, Kastirr I, Gruarin P, et al. Plasticity of human CD4 T cell subsets. Front Immunol 2014;5:630.

12. Vega-Avelaira D, Ballesteros JJ, López-García JA. Inflammation-induced hyperalgesia and spinal microglia reactivity in neonatal rats. Eur J Pain 2013;17:1180-8.

13. Rivest $S$. Molecular insights on the cerebral innate immune system. Brain Behav Immun 2003;17:13-9.

14. Van Dam AM, Bauer J, Tilders FJ, Berkenbosch F. Endotoxin-induced appearance of immunoreactive interleukin- 1 beta in ramified microglia in rat brain: A light and electron microscopic study. Neuroscience 1995;65:815-26.

15. Galic MA, Riazi K, Heida JG, Mouihate A, Fournier NM, Spencer SJ, et al. Postnatal inflammation increases seizure susceptibility in adult rats. J Neurosci 2008;28:6904-13.

16. Bartfai T, Sanchez-Alavez M, Andell-Jonsson S, Schultzberg M, Vezzani A, Danielsson E, et al. Interleukin-1 system in CNS stress: Seizures, fever, and neurotrauma. Ann N Y Acad Sci 2007;1113:173-7.

17. Vezzani A, Granata T. Brain inflammation in epilepsy: Experimental and clinical evidence. Epilepsia 2005;46:1724-43.

18. Vezzani A, Baram TZ. New roles for interleukin-1 beta in the mechanisms of epilepsy. Epilepsy Curr 2007;7:45-50.

19. Wu H, Gao Z. Vertigo with dysautonomia and serious allergy: An unusual case of juvenile Ménière's disease. Int J Pediatr Otorhinolaryngol 2015;79:2438-41.

20. Liang H, Xu L, Zhou C, Zhang Y, Xu M, Zhang C. Vagal activities are involved in antigen-specific immune inflammation in the intestine. J Gastroenterol Hepatol 2011;26:1065-71.

21. Yamamoto T, Kodama T, Lee J, Utsunomiya N, Hayashi S, Sakamoto H, et al. Anti-allergic role of cholinergic neuronal pathway via a7 nicotinic ACh receptors on mucosal mast cells in a murine food allergy model. PLoS One 2014;9:e85888. 